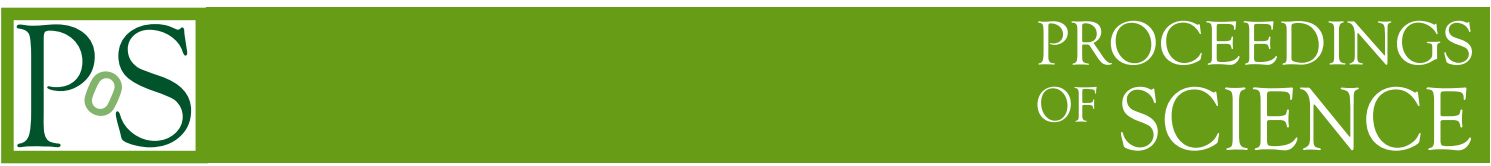

\title{
Electroweak corrections to vector-boson scattering
}

\author{
Benedikt Biedermann, Ansgar Denner*, Mathieu Pellen \\ Julius-Maximilians Universität Würzburg, Würzburg, Germany \\ E-mail: mathieu.pellen@physik.uni-wuerzburg.de, \\ ansgar.dennerephysik. uni-wuerzburg.de, \\ mathieu.pellen@physik.uni-wuerzburg.de
}

\begin{abstract}
We report on a recent calculation of the complete NLO QCD and electroweak corrections to the process pp $\rightarrow \mu^{+} v_{\mu} \mathrm{e}^{+} v_{\mathrm{e}} \mathrm{jj}$, i.e. like-sign charged vector-boson scattering. The computation is based on the complete amplitudes involving two different orders of the strong and electroweak coupling constants at tree level and three different orders at one-loop level. We find electroweak corrections of $-13 \%$ for the fiducial cross section that are an intrinsic feature of the vector-boson scattering process. For differential distributions, the corrections reach up to $-40 \%$ in the phasespace regions explored. At the NLO level a unique separation between vector-boson scattering and irreducible background processes is not possible any more at the level of Feynman diagrams.
\end{abstract}

13th International Symposium on Radiative Corrections (Applications of Quantum Field Theory to

Phenomenology)

25-29 September, 2017

St. Gilgen, Austria

* Speaker. 


\section{Introduction}

The scattering of electroweak (EW) massive vector bosons is a crucial process to be studied at the Large Hadron Collider (LHC). It allows to test the Higgs sector of the Standard Model and provides a very sensitive probe to New Physics. The most promising vector-boson scattering (VBS) channel is the one where to equally charged $\mathrm{W}$ bosons scatter and subsequently decay into two equally charged leptons and two neutrinos. Evidence for VBS in the same-sign WW channel has been reported based on run I data [1,2,3], and the CMS collaboration has observed this process with data from run II [4].

The complete measurable physical process encompassing same-sign VBS is given by $\mathrm{pp} \rightarrow$ $\mu^{+} v_{\mu} \mathrm{e}^{+} v_{\mathrm{e}} \mathrm{jj}$. Parts of the corresponding next-to-leading-order (NLO) corrections have already been computed in the past, such as the NLO QCD corrections for the EW-induced process $[5,6,7]$ and its QCD-induced irreducible background $[8,9,10]$.

In this proceedings contribution we summarize our calculation [11] of the complete NLO corrections to the full process $\mathrm{pp} \rightarrow \mu^{+} v_{\mu} \mathrm{e}^{+} v_{\mathrm{e} j \mathrm{j}}$ featuring in particular surprisingly large NLO EW corrections $[12,13]$.

\section{Calculating NLO corrections to $\mathbf{p p} \rightarrow \mu^{+} v_{\mu} \mathbf{e}^{+} v_{\mathbf{e}} \mathbf{j j}$}

At the amplitude level, the process $\mathrm{pp} \rightarrow \mu^{+} v_{\mu} \mathrm{e}^{+} v_{\mathrm{e}} \mathrm{jj}$ receives two different types of contributions at leading order (LO), pure EW contributions of order $\mathscr{O}\left(g^{6}\right)$ and QCD-induced contributions of order $\mathscr{O}\left(g_{\mathrm{s}}^{2} g^{4}\right)$, where $g$ and $g_{\mathrm{s}}$ are the EW and strong coupling constants, respectively. In Fig. 1 we show some tree-level diagrams for the partonic sub-process u $\overline{\mathrm{d}} \rightarrow \mu^{+} v_{\mu} \mathrm{e}^{+} v_{\mathrm{e}} \overline{\mathrm{u}}$. The $t$-channel diagram on the left illustrates the characteristic VBS topology of two W bosons with space-like momenta that scatter into two $\mathrm{W}$ bosons with time-like momenta. The $s$-channel diagram in the middle contributes to the irreducible EW background; it actually features triple gauge-boson production. Finally, the diagram on the right-hand side provides an example of a QCD-induced contribution. Thus, at the level of the cross section three gauge-invariant contributions exist: the pure EW contri-
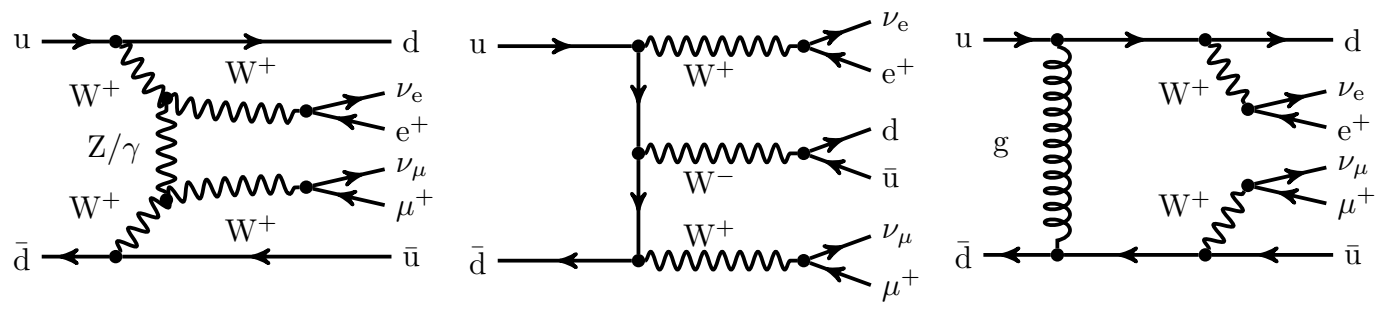

Figure 1: Sample tree-level diagrams contributing to the process $\mathrm{pp} \rightarrow \mu^{+} v_{\mu} \mathrm{e}^{+} v_{\mathrm{e}} \mathrm{jj}$.

bution of order $\mathscr{O}\left(\alpha^{6}\right)$, the QCD-induced contribution of order $\mathscr{O}\left(\alpha_{\mathrm{s}}^{2} \alpha^{4}\right)$, and the interferences of the order $\mathscr{O}\left(\alpha_{\mathrm{s}} \alpha^{5}\right)$. Owing to the colour structure, the interferences occur only in partonic channels that involve contributions of two different kinematic channels $(s, t, u)$. In our calculation we include all contributions that belong to the hadronic process $\mathrm{pp} \rightarrow \mu^{+} v_{\mu} \mathrm{e}^{+} v_{\mathrm{e} j \mathrm{j}}$ at tree and one-loop order. 
The NLO corrections involve contributions of orders: $\mathscr{O}\left(\alpha^{7}\right), \mathscr{O}\left(\alpha_{\mathrm{s}} \alpha^{6}\right), \mathscr{O}\left(\alpha_{\mathrm{s}}^{2} \alpha^{5}\right)$, and $\mathscr{O}\left(\alpha_{\mathrm{s}}^{3} \alpha^{4}\right)$. The contributions of order $\mathscr{O}\left(\alpha^{7}\right)$ furnish the NLO EW corrections to the EW-induced LO process [12]. The order $\mathscr{O}\left(\alpha_{\mathrm{s}}^{3} \alpha^{4}\right)$ contributions are the QCD corrections to the QCD-induced process $[8,10]$. For the orders $\mathscr{O}\left(\alpha_{\mathrm{s}} \alpha^{6}\right)$ and $\mathscr{O}\left(\alpha_{\mathrm{s}}^{2} \alpha^{5}\right)$, a simple separation into QCD and EW corrections is not possible any more. For instance, the order $\mathscr{O}\left(\alpha_{\mathrm{s}} \alpha^{6}\right)$ contains QCD corrections to the EW-induced process $[5,6,7,10]$ as well as EW corrections to the $\mathrm{LO}$ interference. In fact, the virtual corrections of order $\mathscr{O}\left(g_{\mathrm{s}}^{2} g^{6}\right)$ involve diagrams that can be interpreted as EW correction to the QCD-induced process or as QCD correction to the EW-induced process (see diagram in the middle of Fig. 2). Therefore, both processes cannot be separated any more once the full NLO corrections are included.
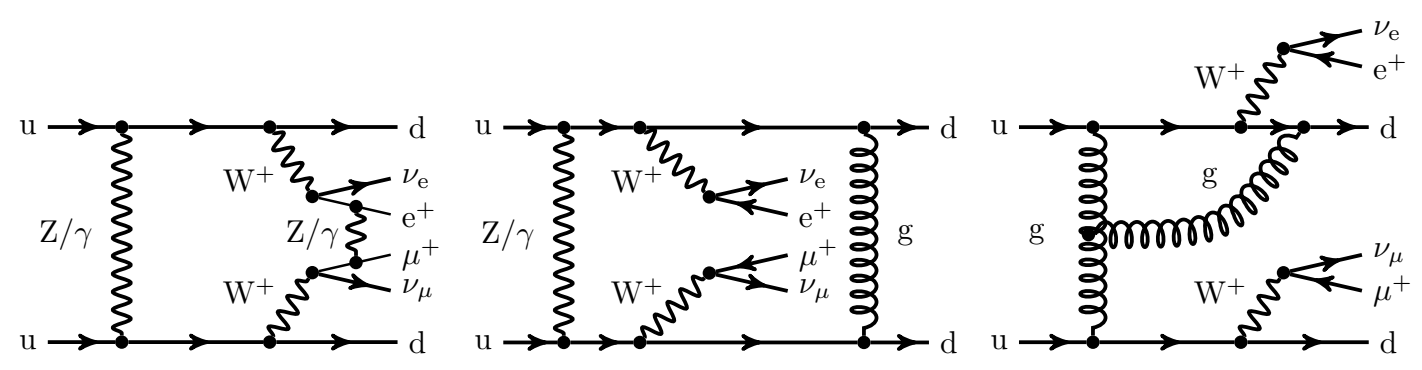

Figure 2: One-loop level diagrams contributing to the process $\mathrm{pp} \rightarrow \mu^{+} v_{\mu} \mathrm{e}^{+} v_{\mathrm{ejj}}$.

Some contributing one-loop diagrams are shown in Fig. 2. The diagram on the left-hand side of order $\mathscr{O}\left(g^{8}\right)$ involves an 8-point function, the one in the middle is of order $\mathscr{O}\left(g_{\mathrm{s}}^{2} g^{6}\right)$ and the one on the right hand-side of order $\mathscr{O}\left(g_{\mathrm{s}}^{4} g^{4}\right)$. All tree-level and one-loop matrix elements have been obtained with ReCOLA [14, 15] in combination with ColLIER $[16,17]$. Throughout, the complex-mass scheme $[18,19]$ is used.

To handle the IR divergences (of QCD and QED origin) in the real NLO corrections the dipole-subtraction method for QCD [20] and its extension to QED [21] have been employed. The colour-correlated matrix elements needed for the subtraction procedure are obtained directly from RECOLA. The QCD radiation of type $\mathrm{pp} \rightarrow \mu^{+} v_{\mu} \mathrm{e}^{+} v_{\mathrm{e}} \mathrm{jjj}$ includes both gluon radiation from any coloured particle as well as quark/anti-quark radiation from contributions with $\mathrm{g} \overline{\mathrm{q}}$ and gq initial states. Similarly, real radiation from photon-induced contributions of the type $\gamma \mathrm{q} / \gamma_{\bar{q}} \rightarrow$ $\mu^{+} v_{\mu} \mathrm{e}^{+} v_{\mathrm{e}} \mathrm{jjj}$ contributes at the orders $\mathscr{O}\left(\alpha^{7}\right), \mathscr{O}\left(\alpha_{\mathrm{s}} \alpha^{6}\right)$, and $\mathscr{O}\left(\alpha_{\mathrm{s}}^{2} \alpha^{5}\right)$. The photon-induced corrections are at the level of $2 \%$ and originate mainly from the contributions of order $\mathscr{O}\left(\alpha^{7}\right)$. We have computed these contributions separately but do not include them in the results shown below. Results for the photon-induced corrections can be found in Ref. [22].

\section{Numerical results}

The input parameters and the selection cuts for the numerical analysis can be found in Ref. [11] and are inspired by the experimental searches for the VBS process [1, 3, 4]. For the centre-of-mass energy we have chosen $13 \mathrm{TeV}$. The renormalisation and factorisation scales are set dynamically to

$$
\mu_{\mathrm{ren}}=\mu_{\mathrm{fac}}=\sqrt{p_{\mathrm{T}, \mathrm{j}_{1}} p_{\mathrm{T}, \mathrm{j}_{2}}}
$$




\begin{tabular}{|l||c|c|c||c|}
\hline Order & $\mathscr{O}\left(\alpha^{6}\right)$ & $\mathscr{O}\left(\alpha_{\mathrm{s}} \alpha^{5}\right)$ & $\mathscr{O}\left(\alpha_{\mathrm{s}}^{2} \alpha^{4}\right)$ & Sum \\
\hline$\sigma_{\mathrm{LO}}[\mathrm{fb}]$ & $1.4178(2)$ & $0.04815(2)$ & $0.17229(5)$ & $1.6383(2)$ \\
\hline
\end{tabular}

Table 1: Fiducial cross section at LO for the process $\mathrm{pp} \rightarrow \mu^{+} v_{\mu} \mathrm{e}^{+} v_{\mathrm{e}} \mathrm{jj}$ in femtobarn. The statistical uncertainty from the Monte Carlo integration on the last digit is given in parenthesis.

\begin{tabular}{|l||c|c|c|c||c|}
\hline Order & $\mathscr{O}\left(\alpha^{7}\right)$ & $\mathscr{O}\left(\alpha_{\mathrm{s}} \alpha^{6}\right)$ & $\mathscr{O}\left(\alpha_{\mathrm{s}}^{2} \alpha^{5}\right)$ & $\mathscr{O}\left(\alpha_{\mathrm{s}}^{3} \alpha^{4}\right)$ & Sum \\
\hline \hline$\delta \sigma_{\mathrm{NLO}}[\mathrm{fb}]$ & $-0.2169(3)$ & $-0.0568(5)$ & $-0.00032(13)$ & $-0.0063(4)$ & $-0.2804(7)$ \\
\hline$\delta \sigma_{\mathrm{NLO}} / \sigma_{\mathrm{LO}}[\%]$ & -13.2 & -3.5 & 0.0 & -0.4 & -17.1 \\
\hline
\end{tabular}

Table 2: Absolute and relative NLO corrections for the process pp $\rightarrow \mu^{+} v_{\mu} \mathrm{e}^{+} v_{\mathrm{e}} \mathrm{jj}$ at the orders $\mathscr{O}\left(\alpha^{7}\right), \mathscr{O}\left(\alpha_{\mathrm{s}} \alpha^{6}\right), \mathscr{O}\left(\alpha_{\mathrm{s}}^{2} \alpha^{5}\right)$, and $\mathscr{O}\left(\alpha_{\mathrm{s}}^{3} \alpha^{4}\right)$ and for the sum of all NLO corrections. The statistical uncertainty from the Monte Carlo integration on the last digit is given in parenthesis.

where $p_{\mathrm{T}, \mathrm{j}_{\mathrm{i}}}, i=1,2$, are the transverse momenta of the two jets with largest transverse momenta within the fiducial volume. To determine the scale variation, the central scale (3.1) has been scaled by factors $\xi_{\text {fac }}$ and $\xi_{\text {ren }}$ within the set

$$
\{(1 / 2,1 / 2),(1 / 2,1),(1,1 / 2),(1,1),(1,2),(2,1),(2,2)\} .
$$

The scale variation is determined by the maximum and minimum obtained with these seven values, and the central scale corresponds to $\xi_{\text {fac }}=\xi_{\text {ren }}=1$.

The LO fiducial cross section split into the different orders of coupling constants is presented in Table 1. For the fiducial volume with VBS cuts the EW-induced process is clearly dominating over its irreducible background processes amounting to $87 \%$ of the cross section of the full process $\mathrm{pp} \rightarrow \mu^{+} v_{\mu} \mathrm{e}^{+} v_{\mathrm{ej} j}$. For the scale dependence of the integrated LO cross section we find

$$
\sigma_{\mathrm{LO}}=1.6383(2)_{-9.44(2) \%}^{+11.66(2) \%} \mathrm{fb}
$$

In Table 2, all NLO corrections to the fiducial cross section split into contributions of the different orders in the strong and EW coupling are presented. The total relative NLO corrections, normalised to the sum of all LO contributions to the full process, amounts to $-17.1 \%$. The bulk of the correction with $-13.2 \%$ originates from the EW corrections of order $\mathscr{O}\left(\alpha^{7}\right)$. The order $\mathscr{O}\left(\alpha_{\mathrm{s}} \alpha^{6}\right)$ corrections amount to $-3.5 \%$, while the other contributions are below a per cent. The scale dependence for the NLO cross section is obtained as

$$
\sigma_{\mathrm{NLO}}=1.3577(7)_{-2.7(1) \%}^{+1.2(1) \%} \mathrm{fb}
$$

corresponding to a reduction by a factor five with respect to the LO.

Nonetheless, the LO and NLO uncertainty intervals do not overlap. The reason is that the purely EW contributions dominate the $\mu^{+} v_{\mu} \mathrm{e}^{+} v_{\mathrm{e}} \mathrm{jj}$ final state, and the NLO EW corrections to VBS represent a large fraction of the NLO corrections. These corrections simply shift the prediction without affecting the size of the scale variation band. While missing higher-order QCD corrections 
can be estimated via scale variations this is not the case for higher-order EW corrections in the on-shell scheme. A conservative estimate for the higher-order EW corrections is provided by the square of the relative EW NLO correction.

The origin of the large EW corrections to the fiducial cross section has been elucidated in Ref. [12]. Using the double-pole approximation and the effective vector-boson approximation for the matrix elements and in addition the high-energy logarithmic approximation for the EW corrections, the large EW corrections can be reproduced. This analysis showed that the EW corrections are large in comparison to those of other LHC processes owing to the relatively high intrinsic energy scale of VBS, the large EW charges of the W bosons, and the relatively weak cancellation between leading and subleading EW logarithms.

The contributions to different coupling orders in the NLO corrections to $\mathrm{pp} \rightarrow \mu^{+} v_{\mu} \mathrm{e}^{+} v_{\mathrm{ejj}}$ as well as the photon-induced contributions to various distributions have been discussed in Ref. [11]. Here, we merely show some additional results for the dependence of the complete NLO corrections on the factorisation and renormalisation scales in Fig. 3. Corresponding results for other distributions can be found in Ref. [11]. In the upper panels, the complete LO prediction as well as the

(a)
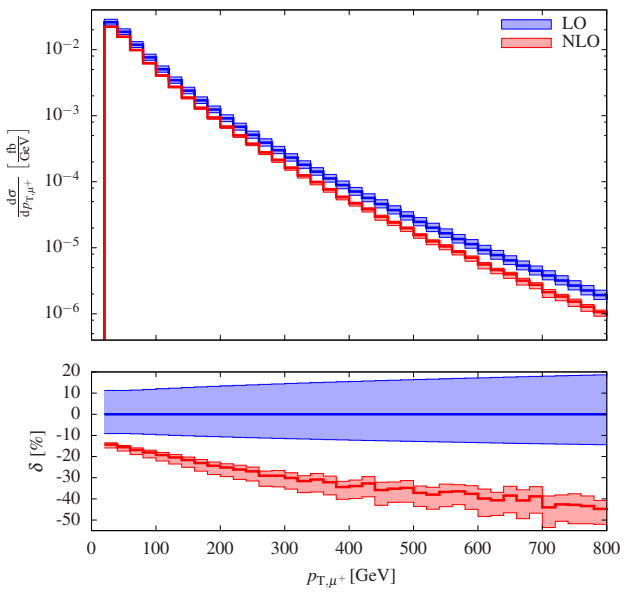

(b)
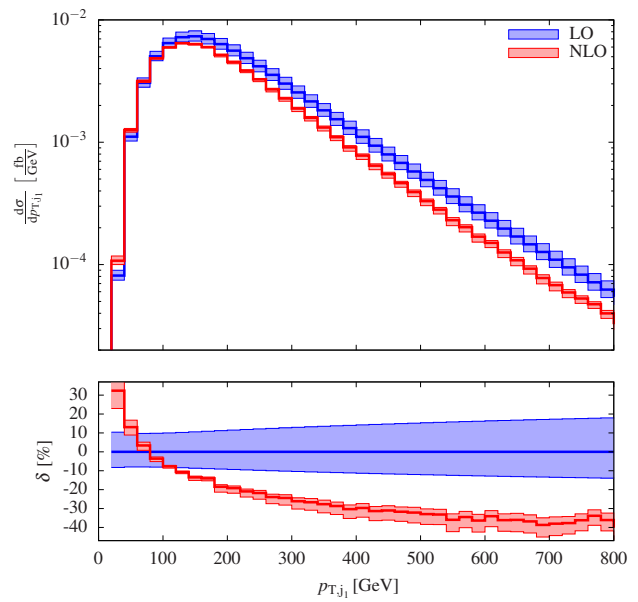

Figure 3: Differential distributions at a centre-of-mass energy $\sqrt{s}=13 \mathrm{TeV}$ at the LHC for $\mathrm{pp} \rightarrow$ $\mu^{+} v_{\mu} \mathrm{e}^{+} v_{\mathrm{e} j \mathrm{j}}$ : (a) transverse momentum of the anti-muon (left), (b) transverse momentum of the hardest jet (right). The upper panels show the sum of all LO and NLO contributions with scale variation. The lower panels show the relative corrections in per cent.

complete NLO prediction are shown. The band is obtained by varying the factorisation and renormalisation scales independently by the factors $\xi_{\text {fac }}$ and $\xi_{\text {ren }}$ with the combinations of (3.2). The complete relative NLO corrections shown in the lower panel are normalised to the LO prediction for the central scale. In Fig. 3a, the distribution in the transverse momentum of the anti-muon is displayed, and the distribution in the transverse momentum of the hardest jet is shown in Fig. $3 \mathrm{~b}$. The scale-uncertainty band decreases significantly by going from LO to NLO for all distributions. The NLO corrections are larger for distributions in leptonic transverse momenta or invariant masses than for those in jet transverse momenta or invariant masses. In general, the scale dependence is larger where the cross section is smaller and the NLO corrections are larger. 


\section{Conclusions}

In this proceedings contribution we have reported on the first calculation of the complete NLO EW and QCD corrections to the process $\mathrm{pp} \rightarrow \mu^{+} v_{\mu} \mathrm{e}^{+} v_{\mathrm{e}} \mathrm{jj}$ including like-sign charged vectorboson scattering (VBS) and its EW- and QCD-induced irreducible background. Using RECOLA, the full LO and NLO matrix elements are used throughout the calculation, including possible offshell, non-resonant, and interference effects. While at LO the EW-induced and the QCD-induced contributions can be unambiguously separated based on the Feynman diagrams and coupling constants, this is not possible anymore at NLO. Hence, for the full process at NLO the VBS process cannot strictly be distinguished from its irreducible background.

The NLO corrections are dominated by large negative EW corrections. For the fiducial cross section, they reach $-13 \%$ of the complete LO contributions and are even significantly more enhanced at the level of differential distributions with up to (minus) $40 \%$ corrections in the kinematical regions explored. These corrections display the typical behaviour of Sudakov logarithms that grow large in the high-energy regime. The dependence on the factorisation and renormalisation scale is significantly reduced upon including NLO corrections. However, this does not provide an estimate of the theoretical uncertainty from missing higher-order EW corrections.

\section{Acknowledgments}

We thank J.-N. Lang and S. Uccirati for supporting the computer program RECOLA and R. Feger for assistance with the Monte Carlo program MoCANLO. We acknowledge financial support by the German Federal Ministry for Education and Research (BMBF) under contract no. 05H15WWCA1 and the German Science Foundation (DFG) under reference DE 623/6-1.

\section{References}

[1] ATLAS Collaboration, G. Aad et al., Evidence for Electroweak Production of $W^{ \pm} W^{ \pm} j$ in $p p$ Collisions at $\sqrt{s}=8 \mathrm{TeV}$ with the ATLAS Detector, Phys. Rev. Lett. 113 (2014) 141803, [arXiv:1405.6241].

[2] ATLAS Collaboration, M. Aaboud et al., Measurement of $W^{ \pm} W^{ \pm}$vector-boson scattering and limits on anomalous quartic gauge couplings with the ATLAS detector, Phys. Rev. D96 (2017) 012007, [arXiv: 1611.02428$]$.

[3] CMS Collaboration, V. Khachatryan et al., Study of vector boson scattering and search for new physics in events with two same-sign leptons and two jets, Phys. Rev. Lett. 114 (2015) 051801, [arXiv:1410.6315].

[4] CMS Collaboration, Observation of electroweak production of same-sign W boson pairs in the two jet and two same-sign lepton final state in proton-proton collisions at $13 \mathrm{TeV}, 2017$. CMS-PAS-SMP-17-004.

[5] B. Jäger, C. Oleari, and D. Zeppenfeld, Next-to-leading order QCD corrections to $W^{+} W^{+} j j$ and $W^{-} W^{-} j$ j production via weak-boson fusion, Phys. Rev. D80 (2009) 034022, [arXiv : 0907.0580 ].

[6] B. Jäger and G. Zanderighi, NLO corrections to electroweak and $Q C D$ production of $W^{+} W^{+}$plus two jets in the POWHEGBOX, JHEP 11 (2011) 055, [arXiv: 1108 . 0864]. 
[7] A. Denner, L. Hošeková, and S. Kallweit, $N L O Q C D$ corrections to $W^{+} W^{+} j j$ production in vector-boson fusion at the LHC, Phys. Rev. D86 (2012) 114014, [arXiv:1209.2389].

[8] T. Melia, K. Melnikov, R. Röntsch, and G. Zanderighi, Next-to-leading order QCD predictions for $W^{+} W^{+} j$ j production at the LHC, JHEP 12 (2010) 053, [arXiv : 1007 . 5313].

[9] T. Melia, P. Nason, R. Röntsch, and G. Zanderighi, $W^{+} W^{+}$plus dijet production in the POWHEGBOX, Eur. Phys. J. C71 (2011) 1670, [arXiv: 1102 . 4846$].$

[10] F. Campanario, M. Kerner, L. D. Ninh, and D. Zeppenfeld, Next-to-leading order QCD corrections to $W^{+} W^{+}$and $W^{-} W^{-}$production in association with two jets, Phys. Rev. D89 (2014) 054009, [arXiv:1311.6738].

[11] B. Biedermann, A. Denner, and M. Pellen, Complete NLO corrections to $W^{+} W^{+}$scattering and its irreducible background at the LHC, JHEP 10 (2017) 124, [arXiv: 1708 . 0 0268].

[12] B. Biedermann, A. Denner, and M. Pellen, Large electroweak corrections to vector-boson scattering at the Large Hadron Collider, Phys. Rev. Lett. 118 (2017) 261801, [arXiv: 1611 . 02951 ].

[13] B. Biedermann, A. Denner, and M. Pellen, NLO electroweak corrections to vector-boson scattering at the LHC, in 25th International Workshop on Deep Inelastic Scattering and Related Topics (DIS 2017) Birmingham, UK, April 3-7, 2017, 2017. arXiv:1708.00646.

[14] S. Actis et al., Recursive generation of one-loop amplitudes in the Standard Model, JHEP 04 (2013) 037, [arXiv:1211.6316].

[15] S. Actis et al., RECOLA: REcursive Computation of One-Loop Amplitudes, Comput. Phys. Commun. 214 (2017) 140-173, [arXiv:1605.01090].

[16] A. Denner, S. Dittmaier, and L. Hofer, COLLIER - A fortran-library for one-loop integrals, PoS LL2014 (2014) 071, [arXiv: 1407 . 0087].

[17] A. Denner, S. Dittmaier, and L. Hofer, COLLIER: a fortran-based Complex One-Loop LIbrary in Extended Regularizations, Comput. Phys. Commun. 212 (2017) 220-238, [arXiv: 1604 . 06792 ].

[18] A. Denner et al., Predictions for all processes $e^{+} e^{-} \rightarrow 4$ fermions $+\gamma$, Nucl. Phys. B560 (1999) 33-65, [hep-ph/9904472].

[19] A. Denner et al., Electroweak corrections to charged-current $e^{+} e^{-} \rightarrow 4$ fermion processes: Technical details and further results, Nucl. Phys. B724 (2005) 247-294, [hep-ph / 0505042 ].

[20] S. Catani and M. H. Seymour, A general algorithm for calculating jet cross-sections in NLO QCD, Nucl. Phys. B485 (1997) 291-419, [hep-ph / 9605323 ]. [Erratum: Nucl. Phys. B510 (1998) 503].

[21] S. Dittmaier, A general approach to photon radiation off fermions, Nucl. Phys. $\mathbf{B 5 6 5}$ (2000) 69-122, [hep-ph/9904440].

[22] B. Biedermann, A. Denner, S. Dittmaier, L. Hofer, and B. Jäger, Next-to-leading-order electroweak corrections to the production of four charged leptons at the LHC, JHEP 01 (2017) 033, [arXiv:1611.05338]. 